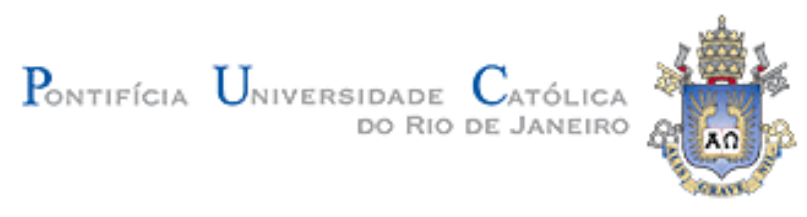

Jordana Luiza Barbosa da Costa Veiga

\begin{abstract}
Análise de Critérios para Aceitação de Enrugamento em Dutos Curvados a Frio
\end{abstract}

DISSERTAÇÃO DE MESTRADO

Dissertação apresentada ao programa de PósGraduação em Engenharia Mecânica da PUC-Rio como requisito parcial para obtenção do título de Mestre em Engenharia Mecânica.

Orientador: José Luiz de França Freire

Rio de janeiro

Outubro de 2008 
Jordana Luiza Barbosa da Costa Veiga

\title{
Análise de Critérios para Aceitação de Enrugamento em Dutos Curvados a Frio
}

Dissertação apresentada como requisito parcial para obtenção do grau de Mestre pelo Programa de Pósgraduação em Engenharia Mecânica do Departamento de Engenharia Mecânica do Centro Técnico Científico da PUC-Rio. Aprovada pela Comissão Examinadora abaixo assinada.

\author{
Prof. José Luiz de França Freire \\ Orientador \\ Pontifícia Universidade Católica do Rio de Janeiro \\ Prof. Carlos Alberto de Almeida \\ Pontifícia Universidade Católica do Rio de Janeiro \\ Prof. Theodoro Antoun Netto \\ Universidade Federal do Rio de Janeiro \\ Dr. Sergio Ricardo Kokay Morikawa \\ PETROBRAS \\ Prof. José Eugenio Leal \\ Coordenador Setorial do Centro \\ Técnico Científico - PUC-Rio
}

Rio de Janeiro, 14 de outubro de 2008 
Todos os direitos reservados. É proibida a reprodução total ou parcial do trabalho sem autorização da autora, do orientador e da universidade.

\section{Jordana Luiza Barbosa da Costa Veiga}

Especializou-se em Engenharia de Dutos pela PUC-Rio em 2007. Graduou-se em Engenharia Mecânica pela Universidade Federal do Rio de Janeiro em 2003 como bolsista de Iniciação Científica do CNPq. Graduou-se em Administração Industrial pelo CEFET-RJ em 2003. É engenheira de equipamentos na Petrobras desde 2005, atuando em Projeto mecânico de tubulações industriais e arranjo de plantas industriais.

Veiga, Jordana Luiza Barbosa da Costa

Análise de critérios para aceitação de enrugamento em dutos curvados a frio / Jordana Luiza Barbosa da Costa Veiga ; orientador: José Luiz de França Freire. - 2008. 206 f. : il. ; $30 \mathrm{~cm}$

Dissertação (Mestrado em Engenharia Mecânica)-Pontifícia Universidade Católica do Rio de Janeiro, Rio de Janeiro, 2008.

Inclui bibliografia

1. Engenharia mecânica - Teses. 2. Curvamento a frio. 3. Enrugamento. 4. Análise de tensões. 5. Fadiga. 6. Transporte dutoviário. 7. Método de elementos finitos. I. Freire, José Luiz de França. II. Pontifícia Universidade Católica do Rio de Janeiro. Departamento de Engenharia Mecânica. III. Título. 


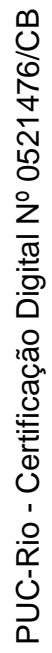

Dedico aos meus Pais, Ana Luiza e José Antônio 


\section{AGRADECIMENTOS}

Agradeço à PUC-Rio pela bolsa de auxílio concedida para realização do mestrado.

Ao Professor Artur Braga pela oportunidade de ter estudado na PUC-Rio.

Aos colegas de trabalho Fábio Marangone, Jorivaldo Medeiros, Ediberto Tinoco, Paulo Roberto, Laudemiro Nogueira, Diógenes Araújo, Rodrigo Hoppe, Rubem Yuan, Edvaldo Casagrande, Jerônimo Loureiro, Djalma Maia, Thiago Mello, Rodrigo Penha, Eduardo Hippert e Gilmar Zacca por toda ajuda e apoio na realização deste trabalho.

Aos colegas de PUC-Rio Maira, Priscilla e Marco pelo incentivo.

Ao colaborador da Smarttech Celso Noronha.

Ao amigo Mauricio Brandão, pelas incansáveis discussões sobre elementos finitos.

Ao engenheiro Marcos Barbosa, da Qualisol, por toda ajuda sobre o processo de curvamento a frio.

À minha Família pelo total apoio, mesmo nos momentos mais difíceis.

E principalmente, ao Professor José Luiz de França Freire pela confiança. 


\section{Resumo}

Veiga, Jordana Luiza Barbosa da Costa; Freire, José Luiz de França (orientador). Análise de Critérios para Aceitação de Enrugamento em Dutos Curvados a Frio. Rio de Janeiro, 2008. 206p. Dissertação de Mestrado - Departamento de Engenharia Mecânica, Pontifícia Universidade Católica do Rio de Janeiro.

Tubos de aço de grande diâmetro, baixa espessura e alta resistência mecânica, possuem tendência à formação de rugas no lado compressivo do curvamento (intrados) ao serem curvados a frio. A presente dissertação descreve os principais códigos de projeto nacionais e internacionais, quanto à presença de rugas provenientes desse tipo de curvamento em dutos, e propõe um ângulo para o qual há a formação do enrugamento. Os códigos internacionais mostram-se conservadores quanto à presença de rugas nos tubos curvados, uma vez que o enrugamento é uma mudança geométrica que, a princípio, gera concentração de tensões e susceptibilidade à ocorrência de falhas por fadiga. Esta dissertação faz uso do método de elementos finitos para modelar a formação do enrugamento e determinar fatores de concentração de tensões, nestas regiões, para carregamentos de pressão interna. Os fatores encontrados são comparados com resultados encontrados na literatura e utilizados no cálculo contra a fadiga por meio de diferentes métodos: Markl, inclinações universais de Manson e ASME seção VIII divisão 2. Neste estudo foram utilizados tubos de aço estrutural API X70 com razão diâmetro espessura (D/t) variando de 20 a 100, modelados por meio do software Abaqus $^{\circledR}$. Foram obtidas curvas com ângulo de $4 \%$ diâmetro e enrugamentos severos, com razão entre a altura da ruga e o diâmetro do tubo (d/D) da ordem de 6,5\% e fator de concentração de tensão chegando a 1,89. Os modelos de tubo enrugado não apresentaram falha na resistência mecânica à pressão interna aplicada, quando esta é suficiente para obtenção de tensão circunferencial nominal equivalente a $100 \%$ do limite de escoamento do material. Os resultados de vida em fadiga para os diferentes métodos aplicados variam de acordo com o método utilizado, mas todos apresentam redução na vida do tubo com presença de enrugamento severo. $\mathrm{O}$ estudo propõe que se utilize para o cálculo da vida em fadiga um procedimento conservador que associa o fator de concentração de tensão determinado por Rosenfeld com o método de cálculo contra a fadiga recomendado pelo código ASME VIII. O estudo sugere ainda, que 
sejam realizadas novas análises de forma a considerar o efeito Bauschinger e a instabilidade à flexão do modelo não avaliados no presente trabalho.

\section{Palavras-chave}

Curvamento a frio; enrugamento; análise de tensões; fadiga; transporte dutoviário; método de elementos finitos. 


\section{Abstract}

Veiga, Jordana Luiza Barbosa da Costa; Freire, José Luiz de França (Advisor). Analysis of Acceptance Criteria of Wrinkles in Pipeline Cold Bends. Rio de Janeiro, 2008. 206p. M. Sc. Dissertation - Departamento de Engenharia Mecânica, Pontifícia Universidade Católica do Rio de Janeiro.

Large diameter, thin walled, high mechanical resistance steel pipe has a tendency to wrinkle on the compressive side (the intrados) of the bend when it is cold bent. This dissertation describes the principal national and international design codes that apply to wrinkling resulting from pipe cold bending, and it proposes an angle at which such wrinkling occurs. The international codes prove to be conservative regarding wrinkling in bent pipe, since a wrinkle is a geometric change, which at first causes a stress concentration and susceptibility to fatigue failure. The dissertation uses the finite element method to model the formation of wrinkling and to determine stress concentration factors in these areas for internal pressure loading. The resulting factors are compared with the results found in the literature and are utilized in calculating fatigue life by means of the following methods: Mark1, Manson's universal inclinations and ASME Section VIII Division 2. In this study API X70 structural pipe with a diameter thickness (D/t) ratio varying from 20 to 100 was utilized, and modeled using Abaqus ${ }^{\circledR}$ software. Bends with an angle of $4 \%$ diameter and severe wrinkling resulted, with a ratio between the peak of the wrinkle and the diameter of the pipe (d/D) of about $6.5 \%$ and a stress concentration factor nearing 1.89. The wrinkled pipe models did not reveal any lack of mechanical resistance to the applied internal pressure when it is sufficient for obtaining a nominal circumferential stress equivalent to $100 \%$ of the yielding limit of the material. The fatigue life results for the different methods varied according to the method that was utilized, but all displayed a reduction in pipe life if there was severe wrinkling. The study proposes a conservative procedure to be utilized for calculating fatigue life. This procedure associates the stress concentration factor determined by Rosenfeld with the method for calculating fatigue recommended by the ASME VIII code. Furthermore, the study suggests that new analyses may be performed in order to consider the Bauschinger effect and the model bend instability, which the study did not evaluated.

\section{Keywords}

Cold bend; wrinkling; stress analysis; pipeline; finite element methods. 


\section{SUMÁRIO}

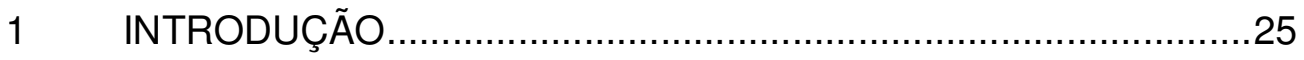

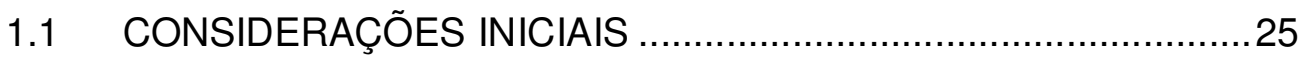

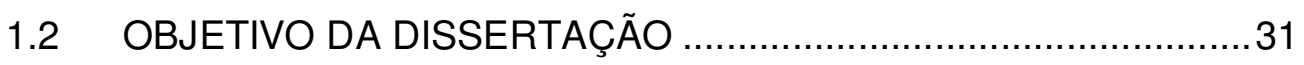

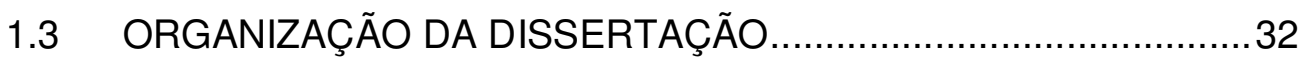

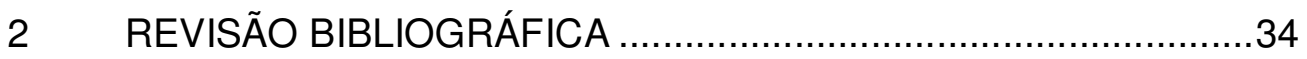

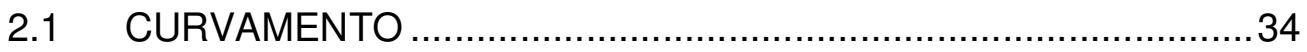

2.1.1 CURVAMENTO A QUENTE POR INDUÇÃO................................34

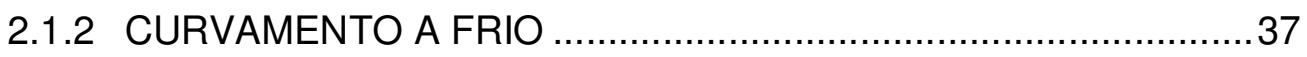

2.2 MÉTODO DE CURVAMENTO A FRIO......................................38

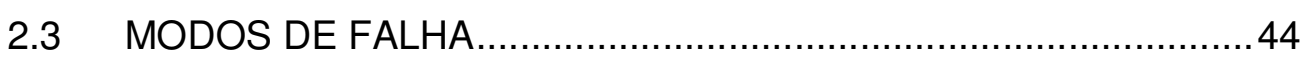

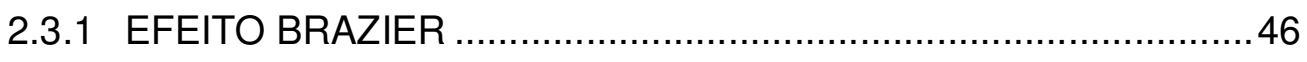

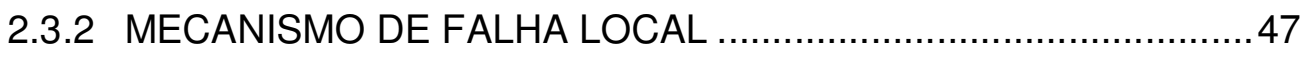

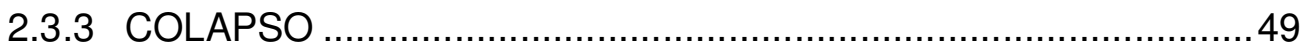

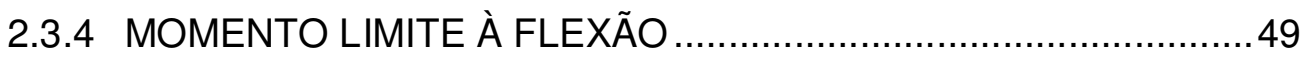

2.4 CRITÉRIOS DE ACEITAÇÃO OU DE REJEIÇÃO PARA

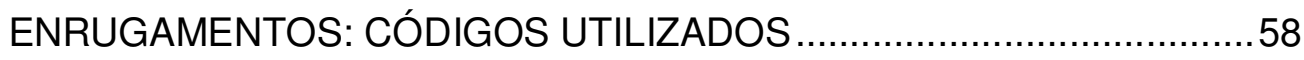

2.4.1 NORMA PETROBRAS N-464 (2004) [9] ………......................59

2.4.2 NBR 12712 (2002) - PROJETO DE SISTEMAS DE TRANSMISSÃO E DISTRIBUIÇÃO DE GÁS COMBUSTÍVEL [46] .........60 2.4.3 CÓDIGO DE REGULAMENTAÇÃO DOS EUA - TITLE 49 [47]

2.4.4 AS 2885.1 (2007) - PIPELINES GAS AND LIQUID PETROLEUM - DESIGN AND CONSTRUCTION [48] ...........................62

2.4.5 DNV OS-F101 (2000) - SUBMARINE PIPELINE SYSTEMS [45]

2.4.6 API 1160 (2001) - MANAGING SYSTEM INTEGRITY FOR HAZARDOUS LIQUID PIPELINE [49]

2.4.7 API 1163 (2005) - IN-LINE INSPECTION SYSTEMS QUALIFICATION STANDARD [50]

2.4.8 ISO 13623 (2000) - PETROLEUM AND NATURAL GAS INDUSTRIES PIPELINE TRANSPORTATION SYSTEMS [51]. 
2.4.9 ASME CODE FOR PRESSURE PIPING, B31..........................68

2.4.9.1ASME B31.3 (2006) - PROCESS PIPING [20] .........................68

2.4.9.2ASME B31.4 (2006) - PIPELINE TRANSPORTATION

SYSTEMS FOR LIQUID HYDROCARBONS AND OTHER LIQUIDS

[53]

2.4.9.3ASME B31.8 (2007) - GAS TRANSMISSION AND DISTRIBUTION PIPING SYSTEMS [54]

2.4.9.4ASME B31.8S (2004) - MANAGING SYSTEM INTEGRITY OF GAS PIPELINES SUPPLEMENT TO ASME B31.8 [55]...................... 71

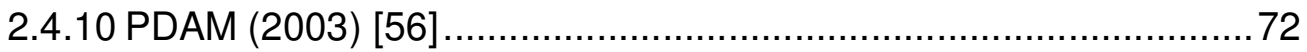

2.4.11 CONCEITOS E DEFINIÇÕES .............................................. 73

2.5 FALHAS DECORRENTES DO ENRUGAMENTO .......................76

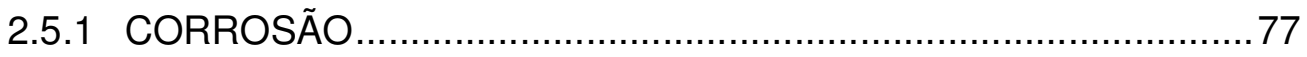

2.5.2 RUPTURA ESTÁTICA ....................................................... 78

2.5.3 COLAPSO PLÁSTICO PROGRESSIVO ................................ 78

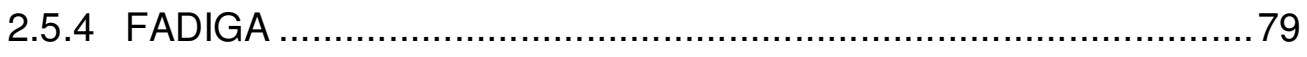

2.5.4.1MÉTODO SN................................................................. 80

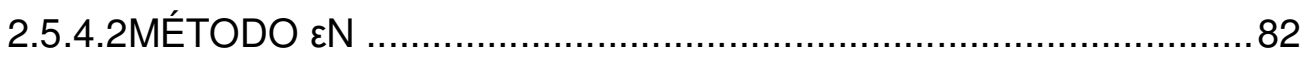

2.5.4.3DANO ACUMULADO ........................................................ 85

2.5.4.4CURVAS DE FADIGA ........................................................ 87

3 ESTUDOS SIMILARES .................................................90

3.1 DEVELOPMENT OF ACCEPTANCE CRITERIA FOR MILD RIPPLES IN PIPELINE FIELD BENDS [21, 22] ..............................90

3.2 LOCAL BUCKLING, STRAIN LOCALIZATION, WRINKLING AND POSTBUCKLING RESPONSE OF LINE PIPE [41] ......................98

3.3 CRITÉRIOS DE ACEITAÇÃO DE ENRUGAMENTOS EM CURVAS DE DUTOS FORMADAS POR CONFORMAÇÃO DE TUBOS RETOS [75]

4 METODOLOGIA .......................................................... 108

4.1 MODELO DE MATERIAL ................................................... 108

4.2 CARACTERÍSTICAS DO TUBO E CASOS PROPOSTOS .........113

4.3 MODELAGEM 


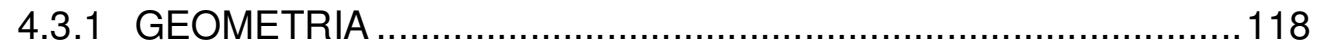

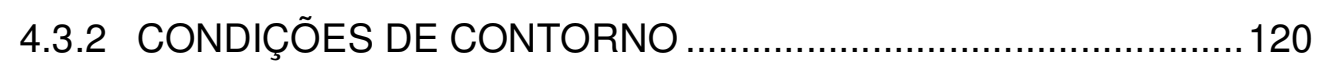

4.3.3 MALHA E ELEMENTOS ............................................................ 123

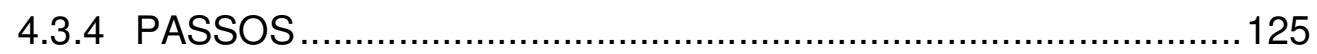

4.4 ANÁLISE DE SENSIBILIDADE DE MALHA .............................126

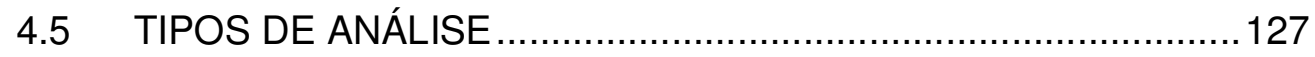

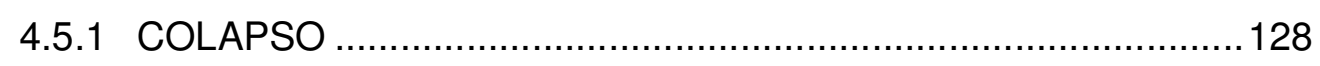

4.5.2 CARREGAMENTO ESTÁTICO …................................................ 129

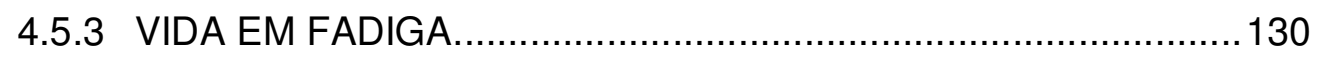

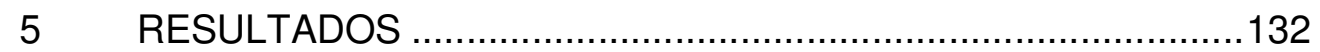

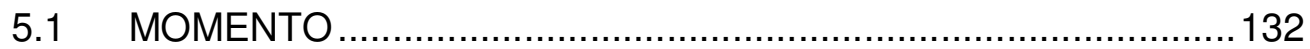

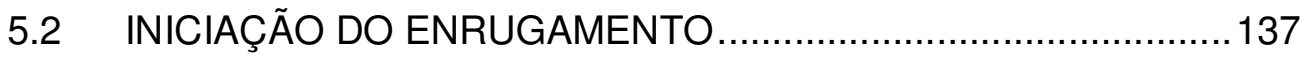

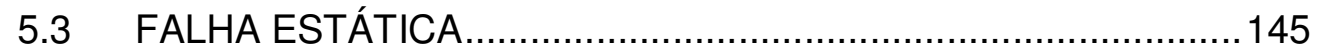

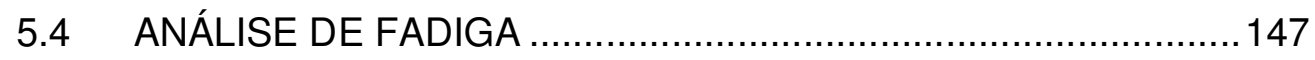

5.5 PROPOSIÇÃO DE METODOLOGIA DE VERIFICAÇÃO DE PERMANÊNCIA DE TUBO COM ENRUGAMENTO............................158

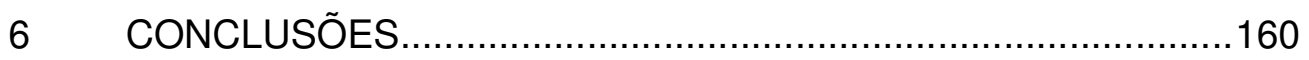

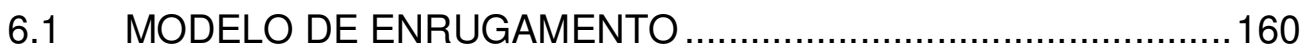

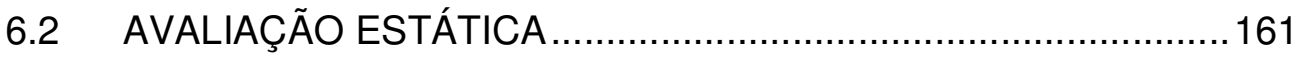

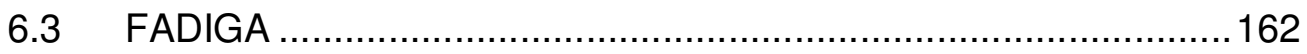

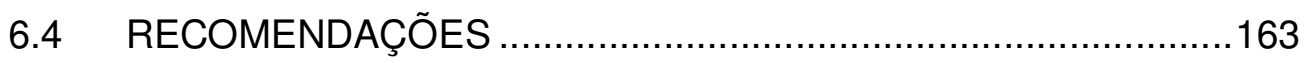

7 REFERÊNCIAS BIBLIOGRÁFICAS .......................................164

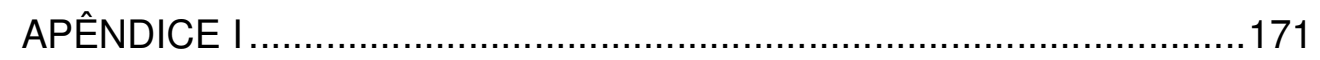

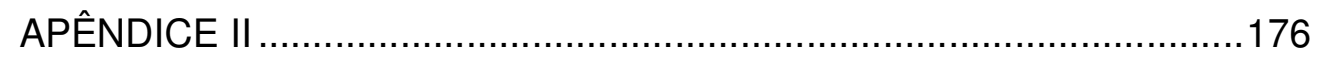

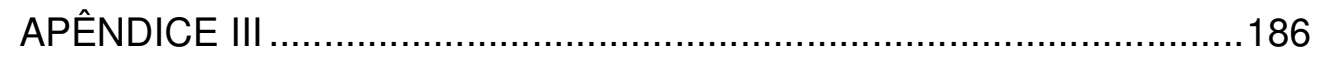

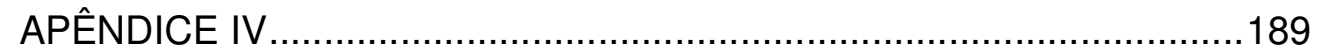

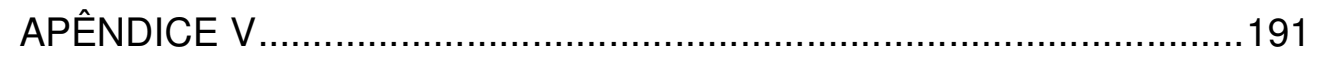

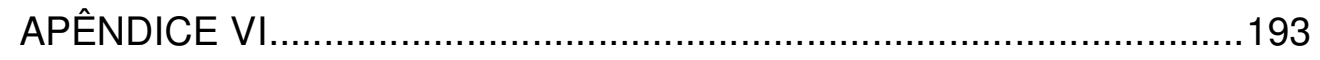

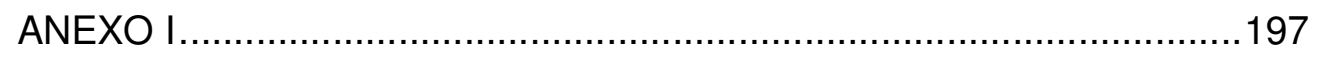

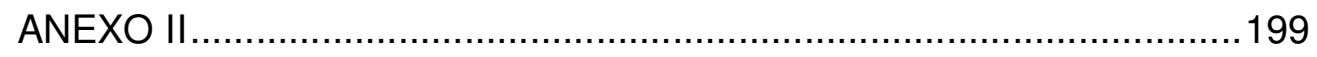




\section{LISTA DE FIGURAS}

Figura 1 - Imagens históricas de processos de curvamento, (a) curvamento a quente e (b) curvamento a frio [1]

Figura 2 - (a) primeiras máquinas de curvamento a frio [1] (b) máquina moderna de curvamento a frio [8]

Figura 3 - Desenho Esquemático de Enrugamento em Tubo

Curvado [18]

Figura 4 - Enrugamentos encontrados em tubos curvados

Figura 5 - Processo de curvamento a quente - (a) curva por indução

a quente em tubo de 48", (b) detalhe da resistência para indução e do resfriamento com jato de água $[23,24]$

Figura 6 - Diagrama esquemático para medição de ondulações [28]......36

Figura 7 - Curvadeira com Tubo Sendo Curvado ................................... 37

Figura 8 - Imperfeições, internas ao tubo, provocadas pelo mandril ........38

Figura 9 - Foto de uma típica curvadeira ......................................... 38

Figura 10 - Esquema de posicionamento do tubo na curvadeira [29]......39

Figura 11 - Detalhe da sela na curvadeira [10] ..................................40

Figura 12 - Esquema do processo de curvamento [7] .........................41

Figura 13 - Figura de um típico mandril utilizado, (a) mandril [29], (b) detalhe das sapatas do mandril [30] e (c) mandril posicionado no tubo [30]

Figura 14 - Figura mostra o imobilizador usado na extremidade do tubo. (a) Imobilizador em uso visto de frente, (b) em uso visto lateralmente e (c) imobilizador.

Figura 15 - Aparelho utilizado na medição do ângulo de cada golpe durante o processo de curvamento (a) posicionado na extremidade do tubo, (b) em uso e (c) mostrador digital.

Figura 16 - Representação da deformação transversal pós-

flambagem [34].

Figura 17- Efeito Brazier. 
Figura 18 - Exemplos de colapso sob flexão pura: (a) Alto D/t Modo diamante de falha, (b) Médio D/t - Dobra local e (c) Baixo D/t Modo de falha difuso [32] .

Figura 19 - Deformação da seção transversal de um tubo sujeito a carregamentos simples [15]

Figura 20 - Exemplo de momento a flexão versus relação de curvatura [15] 50

Figura 21 - Fator de junta soldada [45]

Figura 22- Referência do código AS 2885. (a) para um buckle só, e

(b) dimensões para dois ou mais buckles [48] 63

Figura 23 - Critério para enrugamento - B31.4 [53] .70

Figura 24 - Típico diagrama SN [69].

Figura 25 - Diagrama de Gerber, Goodman e Soderberg [68] .82

Figura 26 - Exemplos do Carregamento Cíclico Simples [66] .86

Figura 27 - Curva de Fadiga para Projeto de Aço Carbono [71]. .87

Figura 28 - Perfil de ruga utilizado por Bilston e Murray [73] 91

Figura 29 - Tensão efetiva, face externa [21] .92

Figura 30 - Relação entre geometria da ruga e SCF (a) para relação d/D e (b) para relação $d / t$...... .94

Figura 31 - Vida em fadiga para enrugamento presente [21] . 95

Figura 32 - Enrugamento permitido recomendado para dutos de transporte de gás e líquidos [21] 96

Figura 33 - Interação solo-estrutura para análise do modelo [41] .99

Figura 34 - Curvas tensão-deformação para tubos carregados axialmente [41] 100

Figura 35 Malha de elementos finitos utilizada na análise [41] 101

Figura 36 - Curvas curvatura-momento para tubos (a) não pressurizados e (b) pressurizados [41] ............................................ 102

Figura 37 - Geometria do tubo e do enrugamento.............................115

Figura 38- Representação do enrugamento criado em FEM.................119

Figura 39 - Modelo para análise de tubo submetido a uma rotação......120

Figura 40 - Deformação devido a erro no processo de modelagem ......120

Figura 41 - Modelo de condição de contorno

Figura 42 - Condições de contorno do modelo no passo de rotação ....122 
Figura 43 - Condições de contorno do modelo no passo de aplicação de pressão.

Figura 44 - Detalhe do enrugamento. (a) Enrugamento após curvamento, (b) enrugamento após alívio

Figura 45 - Imagens de (a) a (f) mostrando a evolução da formação do enrugamento - Caso IV.

Figura 46 - Imagens de (a) a (f) mostrando a evolução da formação do enrugamento - Caso IV - Deformação

Figura 47 - Início enrugamento. (a) Tubo, (b) detalhe para região do enrugamento, (c) detalhe do enrugamento suave visto de frente

Figura 48 - Região C no enrugamento para os nós críticos (a) circunferencial e (b) longitudinal com pressão de $80 \%$ SMYS

Figura 49 - Enrugamento com posicionamento de extensômetros marcados em vermelho, (a) tubo inteiro e (b)vista em corte na região do enrugamento

Figura 50 - Localização da região em estudo para tensão ao longo do diâmetro

Figura 51 - llustração do curvamento pelo fabricante da máquina [30]

Figura 52 - Fotos do processo de curvamento . 202

Figura 53 - Fotos da máquina de curvamento a frio de campo 203

Figura 54 - Mandril penumático 203

Figura 55 - Sequência do curvamento de um tubo 204

Figura 56 - Tubos curvados pelo processo de curvamento a frio de campo. 205

Figura 57 - Tubos curvados com presença de enrugamento. .206 


\section{LISTA DE GRÁFICOS}

Gráfico 1 - Curvas segundo eq. (2) para diferentes relações D/t e diferentes SMYS

Gráfico 2 - Flambagem local sob deslocamento controlado para API

[44] e DNV [45].

Gráfico 3 - Curvas $\varepsilon \times N$ para os aços API X60 (dados experimentais)

e X70 desenvolvida a partir do método das inclinações universais

[75]

Gráfico 4 - Dados obtidos a partir do ensaio de tração com corpo de prova API 5L X70

Gráfico 5 - Dados obtidos corrigidos do ensaio de tração com corpo

de prova API $5 \mathrm{~L}$ X70

Gráfico 6 - Pares de tensão-deformação plástica usados para caracterização do material

Gráfico 7 - Comparação do momento Caso VI para as duas discretizações de malha...

Gráfico 8 - Resultado do Ângulo ( $\left.{ }^{\circ}\right) \times$ Momento ( $\mathrm{x} \mathrm{m}$ ).

Gráfico 9 - Resultado adimensional para Curvatura $X$ Momento.

Gráfico 10 - Para Colapso: Momento X Relação D/t

Gráfico 11 - Relação D/t X máximo momento

Gráfico 12 - Relação D/t X ângulo de máximo momento 136

Gráfico 13 - Apresentação do ângulo de curvamento e final após efeito mola do tubo.

Gráfico 14 - Tensões para o nó externo crítico na tensão circunferencial para o Caso IV

Gráfico 15 - Tensões para o nó interno crítico na tensão longitudinal para o Caso IV

Gráfico 16 - Tensões críticas Caso IV - externo - Nó 104 tensões-

deformações circunferenciais 150

Gráfico 17 - Tensões críticas Caso VI - interno - Nó 2969 tensões-

deformações longitudinais. 
Gráfico 18 - Tensões para o nó externo 70 crítico na tensão circunferencial para o Caso II

Gráfico 19 - Tensões para o nó externo 3556 crítico na tensão longitudinal para o Caso II.

Gráfico 20 - Tensões críticas Caso II - externo - Nó 70 tensõesdeformações circunferenciais.

Gráfico 21 - Tensões críticas Caso II - externo - Nó 3556 tensõesdeformações longitudinais

Gráfico 22 - Tensões para o nó 73 interno crítico na tensão circunferencial para o Caso III

Gráfico 23 - Tensões para o nó 4025 interno crítico na tensão longitudinal para o Caso III.

Gráfico 24 - Tensões críticas Caso III - interno - Nó 73 tensõesdeformações circunferenciais.

Gráfico 25 - Tensões críticas Caso III - interno - Nó 4025 tensõesdeformações longitudinais.

Gráfico 26 - Tensões para o nó 76 externo crítico na tensão circunferencial para o Caso V

Gráfico 27 - Tensões para o nó 4461 interno crítico na tensão longitudinal para o Caso $\mathrm{V}$.

Gráfico 28 - Tensões críticas Caso V - externo - Nó 76 tensões-

deformações circunferenciais.

Gráfico 29 - Tensões críticas Caso V - interno - Nó 4461 tensõesdeformações longitudinais.

Gráfico 30- Tensões para o nó 80 externo crítico na tensão circunferencial para o Caso VI

Gráfico 31- Tensões para o nó 93 interno crítico na tensão longitudinal para o Caso VI

Gráfico 32- Tensões críticas Caso VI - externo - Nó 80 tensõesdeformações circunferenciais.

Gráfico 33- Tensões críticas Caso VI - interno - Nó 93 tensõesdeformações longitudinais.

Gráfico 34 - Tensão longitudinal a cada passo 
Gráfico 35 - Tensão longitudinal ao longo da circunferência quando

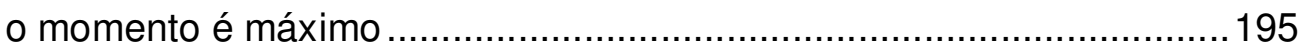

Gráfico 36 - Variação de tensão ao longo do raio do tubo......................196 


\section{LISTA DE TABELAS}

Tabela 1 - Determinação do ângulo máximo da curva ............................71

Tabela 2 - Fatores de concentração de tensões [75] ...........................105

Tabela 3 - Composição Química do Aço API 5L X70 dada pelo código e determinado por ensaios [80].

Tabela 4 - Propriedades mecânicas aço API 5L X70 pela especificação, e obtidos em teste [80] - unidades em MPa (ksi) ...........109

Tabela 5 - Características do Material Modelado API X70

Tabela 6 - Pares tensão-deformação selecionados para caracterização do material - unidade de tensão em MPa.

Tabela 7 - Apresentação dos casos estudados - unidades em mm

(pol.)

Tabela 8 - Valores para raio de curvatura nos modelos

Tabela 9 - Levantamento de casos de enrugamento [85].

Tabela 10 - Dados construtivos para o Abaqus $^{\circledR}$ - unidades em mm (pol.)

Tabela 11 - Descritivo dos passos realizados em FEM

Tabela 12 - Pressões utilizadas - unidades em MPa (ksi)

Tabela 13 - Momento máximo por FEM e da literatura, unidades em

$\mathrm{N} \times \mathrm{m}$.

Tabela 14 - Formação do enrugamento

Tabela 15 - Característica do ângulo das curvas, unidades em grau....139

Tabela 16 - Valores ângulo máximo de curvamento (em graus), altura e comprimento do enrugamento no ponto de máximo momento.

Tabela 17 - Tensões de von Mises máxima encontrada em cada caso.

Tabela 18 - Resultados Geométricos do Enrugamento

Tabela 19 - Relação a/C.

Tabela 20 - Resultado de tensão para cada caso 152

Tabela 21 - Tensão crítica utilizada e seus SCF 154

Tabela 22 - Vida em fadiga para elementos críticos 
Tabela 23 - Características geométricas do enrugamento teste -

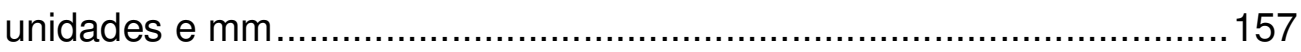

Tabela 24 - Tensão crítica utilizada e seus SCF - complemento à Tabela 20

Tabela 25 - Vida em fadiga para elementos críticos - complemento

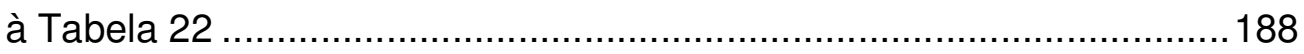

Tabela 26 - Informações CRC-Evans - Máquina 'Centurion' [30] ..........198 


\section{LISTA DE QUADROS}

Quadro 1 - Tradução de Termos .................................................. 74

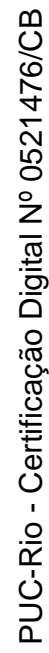

Quadro 2 - Resumo da Aceitação de Enrugamento nos Códigos ...........75 


\section{LISTA DE SÍMBOLOS}
a - Extensão circunferencial
C - Circunferência do tubo
$C_{o}$ - $\quad$ Comprimento de tubo
d - $\quad$ Profundidade da ruga
D - $\quad$ Diâmetro
$D_{\max }$ - $\quad$ Maior valor medido do diâmetro interno ou externo em uma mesma seção

$D_{\min }$ - $\quad$ Menor valor medido do diâmetro interno ou externo em uma mesma seção

$D_{\max A}$ - $\quad$ Maior diâmetro encontrado na seção transversal do tubo

$D_{\min A} \quad$ Menor diâmetro encontrado na seção transversal do tubo

E - $\quad$ Módulo de elasticidade

$E_{\text {ref }}$ - $\quad$ Módulo de elasticidade de referência

$E_{u}-\quad$ Módulo de elasticidade utilizado

FS - $\quad$ Amplitude de tensão circunferencial aplicada

$f_{u}$ - Resistência à ruptura

$f_{y}$ - Tensão de escoamento

$f_{0}$ - Ovalização inicial

$f^{\prime}{ }_{0}{ }^{-} \quad$ Ovalização final

$f_{1}$ - $\quad$ Fator de segurança

$g(\delta)$ - $\quad$ Fator de redução ao colapso

$h$ - $\quad$ Altura pico-vale do enrugamento

$K_{c}$ - $\quad$ Coeficiente de encruamento

$K_{1}-\quad$ Curvatura

L - $\quad$ Comprimento de onda da ruga

$L_{t}$ - $\quad$ Comprimento do tubo

$M_{c}$ - $\quad$ Capacidade de momento

$M_{d}$ - $\quad$ Momento máximo permitido em projeto 


\begin{tabular}{|c|c|}
\hline$M_{p}-$ & Momento plástico do material \\
\hline$M_{0}-$ & Momento \\
\hline$n_{c}-$ & Expoente de encruamento \\
\hline$n_{i}-$ & Número de ciclos aplicados \\
\hline$N-$ & Número de ciclos \\
\hline$N_{i}^{-}$ & Vida do material \\
\hline$P-$ & Limite de proporcionalidade \\
\hline$P_{i}-$ & Pressão interna \\
\hline$P_{c}-$ & Pressão de colapso \\
\hline$P_{0}-$ & Pressão externa \\
\hline$R-$ & Raio externo \\
\hline$R a-$ & Razão entre tensão mínima e máxima \\
\hline$R A-$ & Redução de área \\
\hline$R_{c}-$ & Raio de curvamento \\
\hline $\mathrm{RN}-$ & Número de rugas formadas \\
\hline$S-$ & Tensão circunferencial máxima de operação \\
\hline$S_{a}-$ & Amplitude admissível da componente alternada de tensão \\
\hline$S_{a l t}-$ & Amplitude de tensão encontrada \\
\hline$S C F-$ & Fator de concentração de tensão \\
\hline$S C F_{F E M}-$ & $\begin{array}{l}\text { Fator de concentração de tensão obtido pelo método } \\
\text { nitos }\end{array}$ \\
\hline
\end{tabular}

$S C F_{R}$ - $\quad$ Fator de concentração de tensão obtido pela equação de

Rosenfeld
$S_{f}-$
Resistência à fadiga
$S_{f}(N)$ - $\quad$ Reistência à fadiga para um determinado número de ciclos
$S_{i j}$ - Tensões nas direções principais
SMYS - $\quad$ Limite de escoamento mínimo do material
$S_{u}$ - $\quad$ Limite de ruptura de engenharia do material
$t$ - Espessura
$\alpha$ - $\quad$ Ângulo descrito 


\begin{tabular}{|c|c|}
\hline$\alpha_{c}-$ & Fator de fluxo de tensão devido ao encruamento \\
\hline$\alpha_{g w}-$ & Fator de solda \\
\hline$\varepsilon-$ & Deformação de flexão admissível \\
\hline$\varepsilon_{b}-$ & Deformação de flambagem a flexão pura \\
\hline$\varepsilon_{c}-$ & Deformação resistente característica \\
\hline$\varepsilon_{d}-$ & Deformação \\
\hline$\varepsilon_{d c}-$ & Deformação compressiva admissível de projeto \\
\hline$\varepsilon_{e l}-$ & Deformação elástica \\
\hline$\varepsilon_{p l}-$ & Deformação plástica \\
\hline$\varepsilon_{t}-$ & Deformação total \\
\hline$\varepsilon_{v}-$ & Deformação verdadeira \\
\hline$\varepsilon_{1}-$ & Deformação máxima de instalação por cálculo analítico ol \\
\hline \multicolumn{2}{|c|}{ elementos finitos } \\
\hline$\gamma_{m}-$ & Fator de classe de material \\
\hline$\varepsilon_{\text {real }}-$ & Deformação real \\
\hline$\gamma_{S C}-$ & Fator de classe de segurança \\
\hline$\gamma_{\varepsilon}-$ & Fator de deformação resistente \\
\hline$\delta-$ & Ovalização \\
\hline$\sigma_{a}-$ & Tensão alternada \\
\hline$\sigma_{c}-$ & Tensão crítica encontrada pelo método de elementos finitos \\
\hline$\sigma_{m}-$ & Tensão média \\
\hline$\sigma_{\max }-$ & Tensão alternada máxima \\
\hline$\sigma_{\min }-$ & Tensão alternada mínima \\
\hline$\sigma_{\text {real }}-$ & Tensão real \\
\hline$\Delta \varepsilon-$ & Gama de deformações \\
\hline$\Delta \varepsilon_{n}-$ & Gama de deformação nominal \\
\hline$\Delta \varepsilon_{t}-$ & Gama de deformação efetiva total \\
\hline$\Delta \sigma-$ & Gama de tensões \\
\hline$\Delta \sigma_{n}-$ & Gama de tensão nominal \\
\hline
\end{tabular}


$v-\quad$ Coeficiente de Poisson

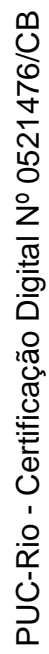

